\title{
Designing Wheelchair-Based Movement Games
}

\author{
KATHRIN M. GERLING, University of Lincoln \\ REGAN L. MANDRYK, MATTHEW MILLER, MICHAEL R. KALYN, \\ and MAX BIRK, University of Saskatchewan \\ JAN D. SMEDDINCK, University of Bremen
}

People using wheelchairs have access to fewer sports and other physically stimulating leisure activities than nondisabled persons, and often lead sedentary lifestyles that negatively influence their health. While motionbased video games have demonstrated great potential of encouraging physical activity among nondisabled players, the accessibility of motion-based games is limited for persons with mobility disabilities, thus also limiting access to the potential health benefits of playing these games. In our work, we address this issue through the design of wheelchair-accessible motion-based game controls. We present KINECT Wheels , a toolkit designed to integrate wheelchair movements into motion-based games. Building on the toolkit, we developed Cupcake Heaven, a wheelchair-based video game designed for older adults using wheelchairs, and we created Wheelchair Revolution, a motion-based dance game that is accessible to both persons using wheelchairs and nondisabled players. Evaluation results show that KINECT Wheels can be applied to make motion-based games wheelchair-accessible, and that wheelchair-based games engage broad audiences in physically stimulating play. Through the application of the wheelchair as an enabling technology in games, our work has the potential of encouraging players of all ages to develop a positive relationship with their wheelchair.

Categories and Subject Descriptors: K.4.2 [Computers and Society]: Social Issues-Assistive technologies for people with disabilities, Handicapped persons/special needs; K.8.0 [Personal Computing]: GeneralGames

General Terms: Design, Experimentation

Additional Key Words and Phrases: Video games, motion-based games, older adults, persons with mobility disabilities, accessibility, assistive devices

\section{ACM Reference Format:}

Kathrin M. Gerling, Regan L. Mandryk, Matthew Miller, Michael R. Kalyn, Max Birk, Jan D. Smeddinck. 2015. Designing wheelchair-based movement games. ACM Trans. Access. Comput. 6, 2, Article 6 (March 2015), 23 pages.

DOI: http://dx.doi.org/10.1145/2724729

\section{INTRODUCTION}

People using wheelchairs have access to fewer sports and other physically stimulating leisure activities than nondisabled persons [Rimmer et al. 2004], with recent reports indicating that more than half of adults using wheelchairs in the United States do not engage in any physical activity [U.S. Department of Health and Human Services 2010]. Sedentary lifestyles and a lack of physical exercise have negative health implications

This work is supported by funding from NSERC, SURFNET, and the GRAND NCE.

Authors' addresses: K. M. Gerling, University of Lincoln, Brayford Pool, Lincoln LN6 7TS, United Kingdom; R. L. Mandryk, M. Miller, M. R. Kalyn, and M. Birk, University of Saskatchewan, 110 Science Place, Saskatoon SK S7N 5C9, Canada; J. D. Smeddinck, University of Bremen, Bibliothekstraße 1, 28359 Bremen, Germany.

Permission to make digital or hard copies of part or all of this work for personal or classroom use is granted without fee provided that copies are not made or distributed for profit or commercial advantage and that copies show this notice on the first page or initial screen of a display along with the full citation. Copyrights for components of this work owned by others than ACM must be honored. Abstracting with credit is permitted.

To copy otherwise, to republish, to post on servers, to redistribute to lists, or to use any component of this work in other works requires prior specific permission and/or a fee. Permissions may be requested from Publications Dept., ACM, Inc., 2 Penn Plaza, Suite 701, New York, NY 10121-0701 USA, fax +1 (212) 869-0481, or permissions@acm.org.

(c) 2015 ACM 1936-7228/2015/03-ART6 $\$ 15.00$

DOI: http://dx.doi.org/10.1145/2724729 
[Tremblay et al. 2010]; in this context, motion-based video games are a promising design opportunity to combat sedentary lifestyles [Mandryk et al. 2014]. Research results show that such games provide cognitive and physical stimulation [Gao and Mandryk 2011], that they can be designed to be beneficial for diverse audiences, such as older adults [Aarhus et al. 2011], and that they have positive effects on the emotional well-being [Jung et al. 2009] of players. However, results also show that the accessibility of motionbased games for persons using wheelchairs is limited (due to limitations of tracking algorithms and few upper body gestures used as input), and while recent efforts have been made to make camera-based systems more accessible by including the option to track seated players, this still limits the number of input options available, and reduces the view of the player on the upper half of their body rather than recognizing the full person. Therefore, additional considerations are necessary to allow persons using wheelchairs to fully participate in motion-based games and to enable them to obtain their full benefits [Gao and Mandryk 2011].

To explore the use of wheelchair-based input in motion-based games, we implemented the KINECT Wheels toolkit [Gerling et al. 2013], which allows us to track wheelchair movements using the Microsoft Kinect sensor. Using KINECT ${ }^{\text {Wheels }}$, we then evaluated the general feasibility of wheelchair-based game input in a controlled study. Building on the results of this initial study, we created two wheelchair-based games with the goal of exploring the accessibility of wheelchair-based game controls among different groups of participants. The first game, Cupcake Heaven, is a motion-based video game designed for older adults that integrates basic wheelchair movements (moving back and forth); we evaluated the game in a user study with eight older adults regularly using wheelchairs. The second game, Wheelchair Revolution, is a motion-based video game that implements more advanced wheelchair-based input and enables competition between persons using wheelchairs and nondisabled persons. We evaluated the accessibility and player experience in the context of competition with eight younger adults. On a general level, our results show that wheelchair-based games are accessible and enjoyable for broad audiences, and that persons using wheelchairs report a positive player experience when competing with nondisabled peers, suggesting that games are a suitable means of providing physical activity to persons using wheelchairs. This article makes three contributions: (1) we show that wheelchair movements can be recognized and interpreted as input to a game using the KINECT Wheels toolkit; (2) we demonstrate that wheelchair-based interaction is suitable for older and younger adults, and that motion-based games can be designed in a way that makes wheelchair input both accessible and enjoyable; and (3) we highlight design opportunities for wheelchairbased games for applications beyond entertainment, and to foster inclusion of persons using wheelchairs by connecting them with nondisabled peers through play.

Providing stimulation through enjoyable leisure activities is an important step in improving the cognitive, physical, and emotional well-being of persons using wheelchairs. Our research can inform the work of game designers and encourage the creation of motion-based video games that address the specific needs of persons using wheelchairs. On a general level, applying assistive devices as an enabling technology for game play offers the opportunity of encouraging players to develop a positive relationship with their assistive device, focusing on its advantages rather than limitations, and it can be leveraged to foster disability awareness and contribute to the inclusion of people using wheelchairs.

This publication is an extended version of a conference paper that was presented at the 15th ACM SIGACCESS International Conference on Computers and Accessibility (ASSETS'13) [Gerling et al. 2013], and builds on data previously published in the Proceedings of the 2014 CHI Conference on Human Factors in Computing Systems [Gerling et al. 2014]. 


\section{RELATED WORK}

In this section, we provide an overview of previous approaches toward integrating wheelchairs in Human-Computer Interaction (HCI), and we present considerations regarding game accessibility for persons with mobility disabilities, and summarize findings that hold implications for wheelchair-based game design.

\section{1. $\mathrm{HCl}$ Research Involving Wheelchairs}

HCI has explored the use of wheelchairs from different angles. The following section gives an overview of wheelchair interaction, and wheelchair-based game controls.

Wheelchair-Based Interaction. Work in the field of wheelchair-based interaction has a strong focus on accessibility; prior research has primarily focused on how people interact with their electric wheelchairs. Felzer and Freisleben [2002] present HaWCoS, a system that proposes including information on muscle contractions rather than handbased input to navigate the environment using a wheelchair. Likewise, Hinkel [2010] suggests the integration of head movements to control electronic wheelchairs to allow for hands-free interaction with other devices. In contrast to these approaches that aim to make the wheelchair itself more accessible, another line of work focuses on applying the wheelchair as an input device to increase the accessibility of other systems, such as computers. Wobbrock et al. [2004] investigate how joysticks of electric wheelchairs can be applied as text entry solutions to facilitate the interaction process by allowing persons using wheelchairs to apply their wheelchair as the input device, rather than using additional technology.

Wheelchair-Based Game Interaction. Research has also explored the integration of electric wheelchairs as a computer game input device. Rossol et al. [2011] propose a framework that implements electric wheelchairs as a game input device; based on this, they design virtual training scenarios to support rehabilitation and wheelchair training by encouraging people to become more familiar with their assistive device. Hasdai et al. [1998] evaluated this type of virtual environment using electric wheelchairs as the input device, and showed that wheelchair-based game input can improve participants' driving skills. They concluded that wheelchair-based game input can be valuable for supporting wheelchair navigation skills.

In contrast to these approaches that focus on the integration of electric wheelchairs into video games, the GAMEWheels [O'Connor et al. 2000] system allows for the use of manual wheelchairs as an input device. The authors designed a custom metal structure that wheelchairs can be mounted on to track basic wheelchair movements. An evaluation of their system [O'Connor et al. 2000] showed that the system produced significant levels of energy expenditure, creating opportunities for physical activity. Likewise, the project AstroWheelie [Cuzzort and Starner 2008] explores whether wheelchair movements can be tracked by attaching Nintendo Wii Remotes to the wheelchair. However, the two major limitations of these approaches are that they either require customdesigned hardware that is not available to the general public, or that sensors need to be attached to the wheelchair, a process that not all people using wheelchairs might be comfortable with; in addition, some people using wheelchairs might require additional assistance to attach the sensors to their chair. In our work, we address these issues by recognizing wheelchair input with the Microsoft Kinect sensor-a commercially available, low-cost camera-based tracking system that has been shown to be a suitable input device for motion-based video games.

In addition to supporting wheelchair-based input, our system supports body-based gestures to broaden the range of player input available to this particular audience. Research addressing full-body motion-based game controls for persons with mobility 
Table I. Overview of Wheelchair-Based and Body-Based Input Supported by KINECTWheels Gesture 4-Body Input (Lift legs) was included because many older adults in nursing homes use wheelchairs despite maintaining basic ability to stand and walk short distances.

\begin{tabular}{lcc}
\hline & Wheelchair Input & Body Input \\
\hline Gesture 1 & Move forward & Clap hands \\
\hline Gesture 2 & Move backward & Raise left arm \\
\hline Gesture 3 & Turn left & Raise right arm \\
\hline Gesture 4 & Turn right & Lift legs \\
\hline
\end{tabular}

disabilities has the challenge of how to allow for wheelchair-accessible interaction while still providing sufficient levels of physical activity, and enabling persons using wheelchairs to gain access to the same games in which nondisabled persons engage. In this context, researchers need to overcome current limitations of motion-based games by providing additional input gestures to allow players using wheelchairs to fully experience motion-based gaming and receive the exertion-related benefits of motionbased play. Our work aims to address these limitations by combining wheelchair input with upper body gestural game interaction. Using the KINECTWheels toolkit [Gerling et al. 2013], we develop and evaluate Cupcake Heaven-a wheelchair-based video game that features wheelchair-based and gestural game input. Building on these results, we extend our work in the design of the wheelchair-accessible motion-based game Wheelchair Revolution to address the question of whether motion-based games are a suitable means of connecting persons with mobility disabilities and nondisabled persons through play.

\section{STUDY 1: DEVELOPING CAMERA-BASED WHEELCHAIR INTERACTION}

KINECTWheels [Gerling et al. 2013] is a toolkit that facilitates the integration of wheelchair input at the development stage using the Microsoft Kinect sensor. In study 1 , we evaluate the feasibility of wheelchair input using our system, and use the results to inform the design of wheelchair-based game controls for motion-based games.

\subsection{The KINECT ${ }^{\text {Wheels }}$ System}

The KINECT ${ }^{\text {Wheels }}$ system is a software library written in C\# based on the Microsoft Kinect SDK that allows developers to easily and quickly add interactivity to their programs. KINECT ${ }^{\text {Wheels }}$ provides the data from the official Microsoft Kinect SDK, as well as extra gestures that help take advantage of the movements that a wheelchair can make. Data available from the Kinect SDK are all the joint positions of the skeleton and raw image data (color and depth). The new gestures the KINECT ${ }^{\text {Wheels }}$ system provides include the direction the wheelchair is pointing, and a gesture for quick movements to the left and right sides as well as the front and the back (Table I). As shown in Table I, KINECT ${ }^{\text {Wheels }}$ also detects traditional full-body motion-based gestures based on joint locations, including clapping hands, raising the left and right arms, and lifting the legs. Once a gesture is recognized, the toolkit allows for a variety of actions, including sending keystrokes that represent the gestures to the operating system so that thirdparty programs (including those implemented in Flash, OpenGL, and XNA) can be controlled using KINECT Wheels

\subsection{System Evaluation}

To determine whether the KINECT Wheels gestures and their recognition rates are appropriate for game input, we integrated the gesture set into an analysis tool. We were 
interested in both the general suitability of the approach, and potential differences between body-based and wheelchair-based game input.

Gesture Analysis Tool. We created a gesture analysis tool based on KINECTWheels in order to determine whether wheelchair-based input can be applied in an efficient and enjoyable way. Based on prior work on full-body motion-based game interaction for older adults [Gerling et al. 2012], we prompted users to complete wheelchair- and body-based movements (Table I). Instructions were provided through images and descriptions. During the interaction process, performance metrics were recorded. Metrics include gesture type, gesture sequences, completion times, and gesture recognition rates. The analysis tool was implemented in C\#, using the Microsoft Kinect SDK 1.5 and Game Studio 4.0.

Participants and Procedure. Although our goal is to design for people who use wheelchairs on a regular basis (e.g., older adults and people with motor disabilities), we used younger nondisabled participants in this initial study. Our primary interest was in the feasibility of the system (i.e., tracking and recognition accuracy); we did not want to put older adults or people with motor disabilities at risk of injury using a system that had not been evaluated regarding safety concerns and physical demand (e.g., whether users could navigate the wheelchair in the small area visible by the Kinect sensor). Because the metrics of interest in this study are the relative completion time and recognition rate of different gestures, we felt confident that a preliminary investigation with younger nondisabled people would help to guide an informed design that could then be evaluated with special populations. Twelve participants (six female) with an average age of 25.67 years $(S D=4.01)$ took part in the evaluation. All participants were right-handed and reported no motor impairments that could have influenced their ability to navigate a wheelchair. None of the participants had prior experience using a manual wheelchair, but all of them had played motion-based video games, seven having previously used the Kinect sensor. At the beginning of the study, participants filled out a consent form and were asked to provide demographic information. Once participants felt confident using the wheelchair, they were given an overview of the recognition tool. Then, participants were asked to complete two sets of body-based and wheelchair-based input to familiarize themselves with the system. Next, participants were asked to complete two different gesture sets consisting of different combinations of wheelchair-based and body-based input. Finally, participants were asked to complete the NASA-TLX [Vidulich and Tsang 1986] and the ISO 9241-9 questionnaire on device comfort [Douglas et al. 1999] to investigate the cognitive and physical challenges when using a wheelchair as an input device as well as the differences between wheelchairbased and body-based input. The NASA Task Load Index (TLX) includes seven items that are ranked on a sliding scale from 0 to 20 , and focuses on the cognitive, physical, and temporal demand experienced by participants. The ISO 9241-9 questionnaire comprises nine items that are ranked on a 5-point Likert scale, investigating aspects such as the physical effort required to apply an interface. The evaluation ended with open questions on participants' perception of wheelchair-based interaction.

Results-Performance. We tracked performance metrics to evaluate the recognition rates and timing of wheelchair- and body-based gestures. Because the first round was treated as a tutorial to teach participants how to interact with KINECT Wheels, only the last two datasets for each participant (a combination of wheelchair- and body-based input) are included in the analysis. We present descriptive statistics in this section and only perform statistical tests when warranted.

Participants performed a total of 576 gestures; 495 gestures were recognized correctly by the system. Hence, the overall recognition rate was $85.9 \%$, with rates being 
Table II. Tracking Results (Number of Tracked, Missed, Percentage) per Gesture (72 Repetitions per Gesture)

\begin{tabular}{lccc}
\hline Gesture & Tracked & Missed & $\%$ \\
\hline Clap hands & 53 & 19 & 73.6 \\
\hline Raise left arm & 70 & 2 & 97.2 \\
\hline Raise right arm & 68 & 4 & 94.4 \\
\hline Lift legs & 35 & 37 & 48.6 \\
\hline Move forward & 72 & 0 & 100 \\
\hline Move backward & 72 & 0 & 100 \\
\hline Turn left & 63 & 9 & 87.5 \\
\hline Turn right & 62 & 10 & 86.1 \\
\hline
\end{tabular}

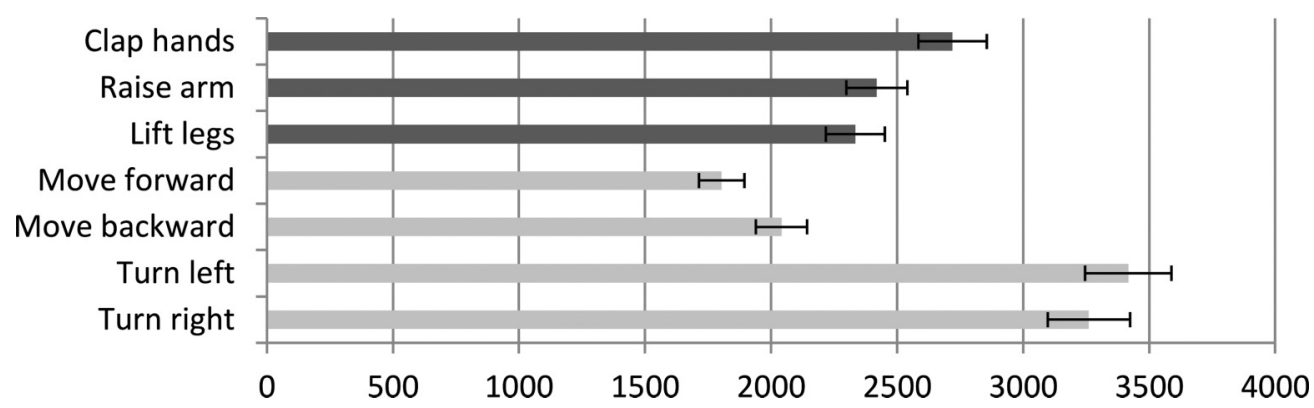

Fig. 1. Average completion time per gesture in milliseconds $(\mathrm{CI}=95 \%)$ for body-based gestures (dark-gray bars) and wheelchair-based gestures (light-gray bars).

lower for body-based gestures (78.5\%) than for wheelchair-based gestures $(93.4 \%)$ (see Table II). Recognition rates for the clapping gesture and leg lift were low due to the technical difficulties of trying to recognize user input in front of participants' bodies; results for all other gestures consistently exceeded an accuracy of $85 \%$. Completing a gesture generally took $2-3 \mathrm{~s}$, with body-based gestures taking $2,471 \mathrm{~ms}$, and wheelchair-based gestures requiring $2,581 \mathrm{~ms}$ (see Figure 1 ).

To investigate whether gesture completion time was influenced by the preceding gesture, we performed a repeated-measures ANOVA and found no difference in gesture completion times (body versus wheelchair) when following a wheelchair-based gesture ( $p=0.081$ ), but that body-based gestures following a body-based gesture are faster $(p=0.000)$ than wheelchair-based gestures that follow a body-based gesture $\left(F_{1,11}=\right.$ $18.9, p=0.001, \eta 2=0.632$ ).

Results-Questionnaires. The results of the NASA-TLX and ISO-9241-9 questionnaires provide further insights into the perceived cognitive load and device comfort. We included a postintervention questionnaire to investigate whether participants enjoyed wheelchair interaction. Comparisons between wheelchair- and body-based gestures were made using paired-samples t-tests.

Descriptive results for all questionnaires are provided in Table III. The composite score [Hart 2006] of the NASA-TLX showed that the cognitive load was significantly higher for wheelchair-based movements than for body-based input $\left(t_{11}=2.61, p=\right.$ 0.024). In addition, we considered the subscale for physical demand, which showed that participants perceived wheelchair-based gestures as more demanding than bodybased input $\left(t_{11}=2.43, p=0.034\right)$, which is supported by the results of the ISO-9241-9 questionnaire $\left(t_{11}=3.46, p=0.005\right)$, particularly when participants were asked about fatigue $\left(t_{11}=2.69, p=0.021\right)$. However, we did not find significant differences for general comfort $\left(t_{11}=-0.23, p=0.082\right)$ and ease of use $\left(t_{11}=-0.69, p=0.504\right)$. 
Table III. Mean (SD) Results for the TLX (20-point scale, composite score 0-120), ISO (5-point scale, composite score 0-45), and Postgame (5-point scale, 5 = strongly agree) Questionnaires

\begin{tabular}{llcr}
\hline & \multicolumn{1}{c}{ Item } & Wheelchair & \multicolumn{1}{c}{ Body } \\
\hline NASA-TLX & Composite score & $51.17(21.35)$ & $28.67(15.35)$ \\
\cline { 2 - 4 } Physical demand & $8.67(4.54)$ & $4.50(3.75)$ \\
\hline PSO-9241-9 & Composite score & $30.08(3.58)$ & $25.42(3.53)$ \\
\cline { 2 - 4 } & General comfort & $3.75(0.97)$ & $3.83(1.12)$ \\
\cline { 2 - 4 } & Ease of use & $4.00(0.74)$ & $4.17(0.72)$ \\
\cline { 2 - 4 } Fatigue & $8.17(3.27)$ & $5.50(1.17)$ \\
\hline Postgame & Fun & $4.17(0.94)$ & $3.50(0.91)$ \\
\hline
\end{tabular}

This suggests that participants consider wheelchair-based gestures more physically challenging, but are still satisfied with overall comfort. This is backed by results regarding fun that show no difference between wheelchair- and body-based input $\left(t_{11}=\right.$ $1.77, p=0.104$ ).

When asked about general problems, participants reported that raising their legs while sitting in the wheelchair was challenging: "Lifting the legs during the body based input was physically demanding, especially while sitting in the wheel chair and trying to establish a good seating position to do the movement." Participants sometimes found it difficult to return to a neutral position after gesture completion: "A large part of the stress of performing the task was trying to get back to the same position before starting the next movement," which corresponds to experimenter observations that were made during the evaluation. In general, comments showed that participants perceived body-based input as more intuitive, but considered wheelchair-based input to be more enjoyable: "Body based input seems to be more natural, and easier to control."- "The wheelchair based input was more fun and engaging than the body based input."

Findings. In this section, we interpret our results, which can inform the design of wheelchair-based game controls. Our results for the KINECT Wheels tracking accuracy show a trade-off between achieving high tracking rates and offering a broad gesture set; both of which can affect player experience. If tracking rates are too low, players may experience frustration, whereas a small gesture set may cause boredom. Our results suggest that body-based input was more problematic than wheelchair-based input, with recognition rates being particularly poor for gestures that were performed in front of the player's body. However, recognition rates for other types of input were acceptable, and the negative effects of lower tracking rates on player experience can be minimized by appropriate design decisions, for example, by applying well-recognized gestures to frequent or crucial game events. Average completion times of around 2.5s per gesture show that participants were able to interact with the system, and that input was fast enough for use in an interactive system. However, designers need to consider time differences between gestures (e.g., moving the wheelchair back and forth is faster than turning it to the sides) and individual players. Our results show that the previous gesture type (wheelchair or body) affects gesture completion time; participants generally required more time when following up on wheelchair-based input as this required them to readjust their hands or deal with movement momentum that had built up. This dependence should be considered when designing input sequences: designers can either combine gestures in a way that increases challenge, or allow users to refocus by introducing sequences of easily combined gestures. Finally, games should consider how to support players when returning to a neutral position after gesture completion. This could be achieved through the inclusion of on-screen menus (i.e., a two-dimensional (2D) minimap indicating the user's location relative to the camera) to help users remain within a certain range of the sensor. 
When interpreting our results, it is important to consider how they would extend to usage by different audiences, for example, younger adults with mobility disabilities, or older adults with age-related mobility impairments. In particular, we expect that some players would require more time to complete gestures than nondisabled younger adults, and that tracking accuracies might be lower given the smaller range of motion of some players, e.g., older adults [Gerling et al. 2012].

\section{STUDY 2: WHEELCHAIR-BASED GAME CONTROLS FOR OLDER ADULTS}

To follow up on the results of our first study, which showed that wheelchair-based interaction is generally safe to use and suited for interactive systems, we apply the results of study 1 in the context of game design for older adults to inform the design of the wheelchair-based game Cupcake Heaven. Using the game, we conduct a second study to validate the feasibility of wheelchair-based input in the context of interaction design for older adults, and explore how the gesture set can be integrated into wheelchair-based video games that are accessible and enjoyable for older adults.

\subsection{Wheelchair Input for Older Adults}

Motion-based game interaction for older adults has unique challenges because of the special characteristics of the target audience that extend beyond mobility disabilities; this section summarizes age-related changes and impairments, as well as motion-based game design for older adults to inform the design of wheelchair-based games.

Age-Related Changes and Impairments. Age-related changes such as decreases in sensory acuity (particularly vision and hearing), and changes in memory and attention, affect the ability of older adults to interact with computers and video games [Czaja and Lee 2006]. Age also leads to a reduction of muscle mass, which causes decrements in strength and stamina, resulting in a lack of movement control and higher reaction and movement times [Ketcham and Stelmach 2001]. Furthermore, older adults are likely to be affected by age-related diseases (e.g., arthritis or orthopedic impairments [Czaja and Lee 2006]), which affect their physical abilities and their capacity to use standard interfaces. Particularly when designing wheelchair-based controls, it is important to understand the impact that age-related decrements may have on older adults' abilities of navigating manual wheelchairs. It is not only important to ensure the general accessibility of interfaces by accommodating a variety of abilities and accounting for age-related changes that influence interaction, but it is also important to ensure that interaction paradigms are safe and do not put older adults at risk of injury from excessive strain.

Motion-Based Game Controls for Older Adults. A growing number of projects focus on the development of accessible motion-based applications for older adults with the goal of providing physical and mental stimulation, e.g., for people living in nursing homes. Full-body motion-based games usually require players to be able to stand without assistance and move around in the room freely. Examples include the virtual dancing environment DanceAlong by Keyani et al. [2005], and motion-based minigames by Rice et al. [2011]. DanceAlong strongly focuses on foot-based input; the minigames proposed by Rice et al. primarily require upper limb interaction. Gerling et al. [2012] present an initial design of a motion-based game that is accessible to older adults who use assistive devices. Older adults are invited to play a gesture-based gardening game that is accessible to people playing either while standing or sitting in a chair.

Based on these results as well as an analysis of the aforementioned age-related changes and design recommendations for full-body motion-based game interaction for older adults [Gerling et al. 2012], we identified issues relevant to wheelchair-based interaction for older adults. 
Easy gesture recall should be supported by full-body input through the implementation of natural gesture mappings, for example, by building on movements that players can relate to real-world actions [Gerling et al. 2012]. In the context of wheelchairbased interaction, integrating the wheelchair in a way that helps participants relate wheelchair input to in-game actions could help facilitate gesture recall. An example of an intuitive mapping is applying the wheelchair in a racing game, encouraging players to move forward to speed up, move back to break, and turning the chair to the sides to make turns in the game.

Adapting to player range of motion [Gerling et al. 2012] is important when designing upper body gestures to go along with wheelchair-based input.

Furthermore, adapting to player strength is important, because the evaluation of KINECT $^{\text {Wheels }}$ showed that wheelchair input is perceived as more physically demanding than body-based input, and older adults experiencing age-related changes may experience difficulties when repeatedly using their upper body to navigate the wheelchair.

Exertion management [Gerling et al. 2012] is another aspect that needs to be considered when integrating wheelchairs into the interaction process. Designers need to keep in mind that wheelchair movements require more effort, and are slower than body-based input; an aspect that has to be considered in the pacing of the game, and when determining the frequency at which certain types of input are required.

Age-inclusive design [Gerling et al. 2012] is necessary to embrace special characteristics of the target audience. Regarding wheelchair-based game interaction for older adults, it is important to keep in mind that many older adults are novice wheelchair users who have limited experience with their assistive device. Designers should account for this aspect by carefully designing wheelchair-based interaction into their games, slowly increasing the difficulty and frequency of input in order to allow players to become more familiar with their assistive device.

In the following section, we will highlight how Cupcake Heaven builds on these design considerations as well as the results of study 1 to provide accessible and safe interaction for institutionalized older adults.

\subsection{The Cupcake Heaven Video Game}

To investigate the feasibility of wheelchair-based game input for older adults, we created Cupcake Heaven (Figure 2), a game in which players are invited to collect candy and pass it on to a girl. To increase the challenge, players have to avoid collecting vegetables, and the girl changes location, either being displayed on the right or left side of the screen. The game implements body-based and wheelchair-based input; it aims to encourage users to move around in their wheelchair. We implemented the game in C\# using the Microsoft Kinect SDK 1.5.1, Microsoft Game Studio 4.0, and the KINECT Wheels toolkit.

Wheelchair-Based Game Input. Based on the results of study 1 and the design requirements in Section 4.1, we identified a set of wheelchair-based and body-based gestures that were well recognized by the system, and that we expected to be easy to learn for older adults. There are two lanes in the game: one on the top of the screen and one on the bottom. Candy and vegetables flow left to right through the top lane and the bottom lane. In order to pick up candy from either of the two lanes, players are asked to move the wheelchair forward and backward, which moves the virtual hand up and down, respectively. Intersecting the hand with a food item automatically picks it up. The game only allows for vertical movements to facilitate simple interaction; players are not expected to adjust their horizontal position to pick up candy. To pass on candy to the child, players have to lift their arm on the side at which the child is currently 


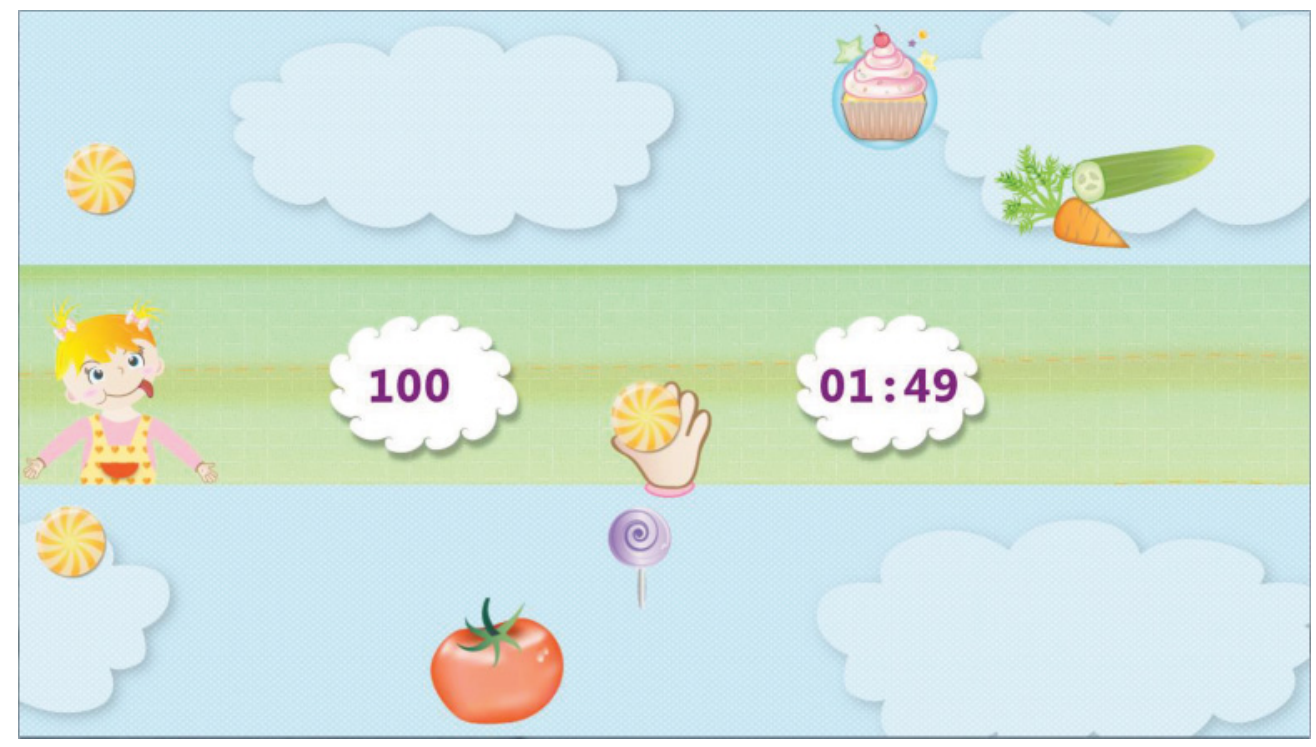

Fig. 2. Overview of Cupcake Heaven.

displayed; this will dispatch the item. If players wish to discard an item that they collected, they have to clap their hands. We decided to implement this gesture using an improved tracking algorithm, because results from Gerling et al. [2012] suggest that clapping is particularly easy for older adults, and because there is little negative impact in the game if the clapping gesture is not quickly recognized. In order to accommodate the various abilities of older adults, the pacing of Cupcake Heaven can be adapted. This includes changing the speed at which candy passes by to allow users to take more time to make a selection, as well as longer availability of the child before she moves to the other side.

\subsection{Evaluation}

In the evaluation, we applied Cupcake Heaven to examine the feasibility of wheelchairbased game input for older adults.

Participants and Procedure. We carried out our study in collaboration with Brightwater Senior Living and Sherbrooke Community Centre in Saskatoon, SK, Canada. Ten older adults participated in the evaluation. Data from two participants were removed from the study (in one case, tracking difficulties were caused by a nonremovable tray mounted on the wheelchair; another participant withdrew after the introduction). The average age of the remaining participants was 75 years $(\mathrm{SD}=5.53$, three males). All participants used manual wheelchairs, with three of them having used a wheelchair for more than 10 years, four having used wheelchairs for 1 to 3 years, and one participant having used a wheelchair for 2 months. Three participants had had a stroke that led to hemiplegia, and one participant was diagnosed with cerebral palsy. Two participants maintained basic ability to stand and walk, but use wheelchairs on a daily basis. After giving consent, participants provided demographic information. Next, participants were given time to familiarize themselves with the wheelchair and the environment, and were introduced to Cupcake Heaven in a short tutorial. Then, participants were asked to play the game for about $10 \mathrm{~min}$, each level of the game lasting $3 \mathrm{~min}$. Finally, participants were asked to complete the NASA-TLX [Hart 2006] questionnaire and 


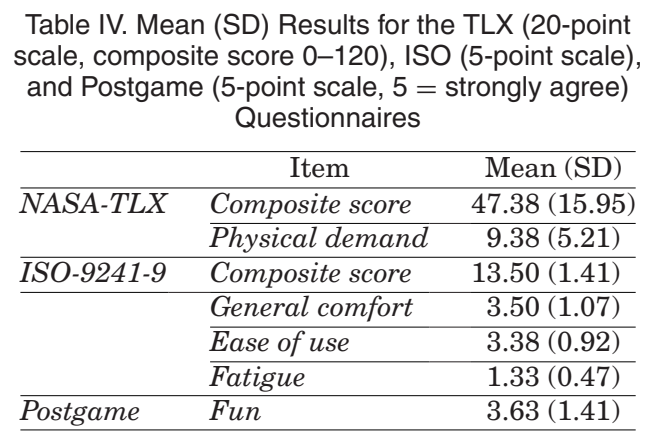

the portions of the ISO 9241-9 [Douglas et al. 1999] questionnaire on device comfort that investigates physical fatigue to assess their experience using the wheelchair as a game input device. In addition, participants were asked to rate their overall experience with the game and answer interview questions on its content, input gestures, and the overall play experience.

Results. Data was analyzed with a focus on the accessibility, usability, and player experience of older adults engaging with the wheelchair-based video game Cupcake Heaven. We organize the presentation of the results by our research questions.

Are wheelchair-based video games suitable for older adults? Our results show that all participants were able to interact with Cupcake Heaven. During each $3 \mathrm{~min}$ level, players interacted with an average of 10.19 ( $\mathrm{SD}=2.77$ ) game items (candy or vegetables), picking them up and either releasing them by lifting their arm, or discarding them by clapping their hands. Out of these items, an average of $2.76(\mathrm{SD}=1.12)$ was fed successfully to the child. Other items were discarded, represented the wrong kind of item (vegetable), or fed at a time when the child was not present. Participants reached an average score of $475(\mathrm{SD}=237.44)$. In terms of player experience, results of the postgame questionnaire show that participants reported positive levels of fun (Table IV). When asked about their detailed experience with the game and their opinions on applying a wheelchair as an input device, participants pointed out that they enjoyed playing games with their wheelchair: "It was fun. I like challenges."- "I enjoyed using my motor skills."- "It was mentally and physically stimulating." Observations support the notion that participants enjoyed the game, with some players getting excited about collecting candy or moving along with the background music.

Can wheelchair-based game controls provide cognitive and physical stimulation for older adults? Findings from our study suggest that wheelchair-based game controls challenge older adults physically and cognitively, but are manageable on an overall level, suggesting that it is possible to strike a balance between challenging and thus engaging players, and keeping players from experiencing undue stress and fatigue.

The results of the NASA-TLX questionnaire (Table IV) show that the cognitive load of older adults interacting with Cupcake Heaven was at an average level, and that it produced a medium level of physical demand. This is supported by results of the ISO 9241-9 questionnaire (Table IV) that show that the system was rated as relatively easy to use and comfortable. In contrast to the results of the NASA-TLX, items of the ISO questionnaire investigating physical fatigue report low levels of physical demand. These findings need to be interpreted along with observations that were made during the study, which contradict subjective ratings for fatigue that were made through the ISO questionnaire; many participants seemed to be challenged by the system and became increasingly tired throughout the gaming session (heavier breathing, 
individual comments on physical exertion). This suggests that wheelchair-based video games have the potential of providing much needed physical exertion for institutionalized older adults. Participants recognized this opportunity and highlighted the benefits of physical activity made accessible through the game. One participant pointed out that the game helped him see the wheelchair in a different light, commenting that the game gave him "the idea that you can use the [wheel]chair for something other than sitting on it." Other participants explicitly commented on using video games to be physically active: "It is good for me [to be active]; I did track and field when I was young, and I played tennis and basketball."- "If you played it for half an hour, you'd get some exercise."

How can our results help inform future projects integrating wheelchair controls into video games for older adults? Observations and questionnaires revealed further insights into how participants interacted with Cupcake Heaven that can help inform future design efforts. While all participants could interact with the system on a basic level, some persons experienced difficulties coordinating multiple movements when moving back and forth, and simultaneously trying to drop off candy. We observed large differences in participants' abilities of controlling their wheelchairs, and the speed at which they were able to navigate. Along these lines, some participants paid close attention to moving their wheelchair, being unable to follow simultaneous in-game events. Sometimes, participants would leave their arm up in the air after successfully discarding an item, triggering undesired input. Some participants needed frequent reminders of possible in-game actions and seemed to experience difficulties coordinating their movements.

In terms of in-game behavior and coping with challenges, some participants showed difficulties remembering to collect candy while avoiding vegetables, sometimes leading to frustration when feeding an item to the child did not trigger the expected response. In contrast, one participant pointed out that she did not find Cupcake Heaven stimulating enough, and that she would have preferred a more complex game: "It wouldn't be a game I'd play very often, I'd find it boring," highlighting that it would be more fun in a multiplayer setting: "It might be more fun to play with somebody else." Another participant explicitly commented on the way input was integrated, stating that she "found it tiring when [she] went back and [the avatar] didn't start moving right away." This outlines a core challenge future research will have to address: Although many institutionalized older adults use wheelchairs, they use the assistive device for a variety of reasons (e.g., short-term recovery from illness, lifelong disabilities, or recent agerelated changes), and are a diverse audience in cognitive and physical abilities, and overall familiarity with their wheelchair.

These results show that wheelchair-based game controls are generally accessible to older adults, and that games can provide an enjoyable activity that can be leveraged to provide this audience with an opportunity to become more familiar with their assistive device; however, there are challenges in game design and controller mappings specific to older adults that must be taken under consideration.

\section{STUDY 3: ENGAGING NONDISABLED PERSONS AND PERSONS USING WHEELCHAIRS IN GAME-BASED COMPETITION}

Given the success of deploying wheelchair game controls with an audience of older adults, we were also interested in exploring how to engage younger persons using wheelchairs due to mobility disabilities in motion-based play. Although many of the technical challenges of recognizing input are similar between these two audiences, the resulting player experience for these two groups needs to be explored individually. For example, the limits in the speed of gesture recognition were not a problem in Cupcake Heaven; however, this might be an issue when creating a game for a younger audience familiar with fast-paced console game play. 
To explore wheelchair-based game controls for a younger audience, we created Wheelchair Revolution, a motion-based dancing game that builds on game mechanics similar to those of Dance Dance Revolution-one of the most successful examples of commercially available motion-based games [Höysniemi 2006]. Wheelchair Revolution can either be played using a manual wheelchair, or a dance mat for game input. As Wheelchair Revolution is intended to be a multiplayer competitive game (similar to Dance Dance Revolution), we also need to balance for players of different skill or ability to compete against each other (called player balancing). In addition, the potential use of two different input techniques if a player using a wheelchair competed against a nondisabled player (and thus used the wheelchair and a dance mat, respectively, to control the game) means that off-the-shelf balancing approaches might not be sufficient. Thus, we also created and applied balancing strategies (that are described in detail and evaluated in Gerling et al. [2014]) to mitigate differences caused by the different input modes, and to accommodate players of all abilities. Here, in our analysis of results, we focus on the experience of the player using wheelchair-based input and associated challenges regarding accessibility and game design to evaluate wheelchair-based game control for a younger audience.

\subsection{Overview of Wheelchair Revolution}

As discussed in Gerling et al. [2014], Wheelchair Revolution is played by making input in sync with music. Along with the rhythm of each song, a number of arrows are displayed on the screen (Figure 4) that-depending on input device-need to be matched by player steps on the dance mat or wheelchair movements tracked through KINECT ${ }^{\text {Wheels }}$. Arrows move into the screen area from the top and need to be matched by player input once they reach the targeting area at the bottom of the screen (Figure 4). The game supports four different input types: up (stepping to the front or wheeling forward), down (stepping back or wheeling back), left (stepping left or turning the wheelchair to the left), and right (stepping right or turning the wheelchair to the right). Scores are calculated based on player timing; accuracy is determined based on how close input was made to the targeting area (miss, okay, good, and perfect). In two-player mode, competition is created by inviting players to compete based on the same song; a split-screen approach is applied with each contestant being assigned one side of the screen that displays arrows and feedback.

\subsection{Game Input}

As described in Gerling et al. [2014], Wheelchair Revolution builds on Microsoft XNA Game Studio 4.0 and the Microsoft Kinect SDK; the game is implemented in C\#. Wheelchair-based player input is integrated using the KINECT Wheels toolkit that we introduced and evaluated in Section 3.

Foot-Based Game Input. Foot-based input is recorded using a dance mat that is commonly used for player input in Dance Dance Revolution. Input is made on a grid (Figure 3 ) that is labeled with arrows matching those displayed in the game (Figure 4). Players place themselves in the center of the mat; once the game starts, they can tap cells with their feet to make input.

Wheelchair-Based Game Input. Wheelchair input builds on the KINECT ${ }^{\text {Wheels }}$ toolkit and the evaluation results of study 1 described in Section 3. It is designed similarly to foot-based input; player movements are tracked using a grid-style approach where the location of the wheelchair determines which arrow is triggered: if the wheelchair is moved toward the screen, an up-step is registered; if the wheelchair is moved back, a down-step is triggered. Likewise, turning to the sides results in triggering a step to the respective side. We used this approach to create wheelchair-based game input 


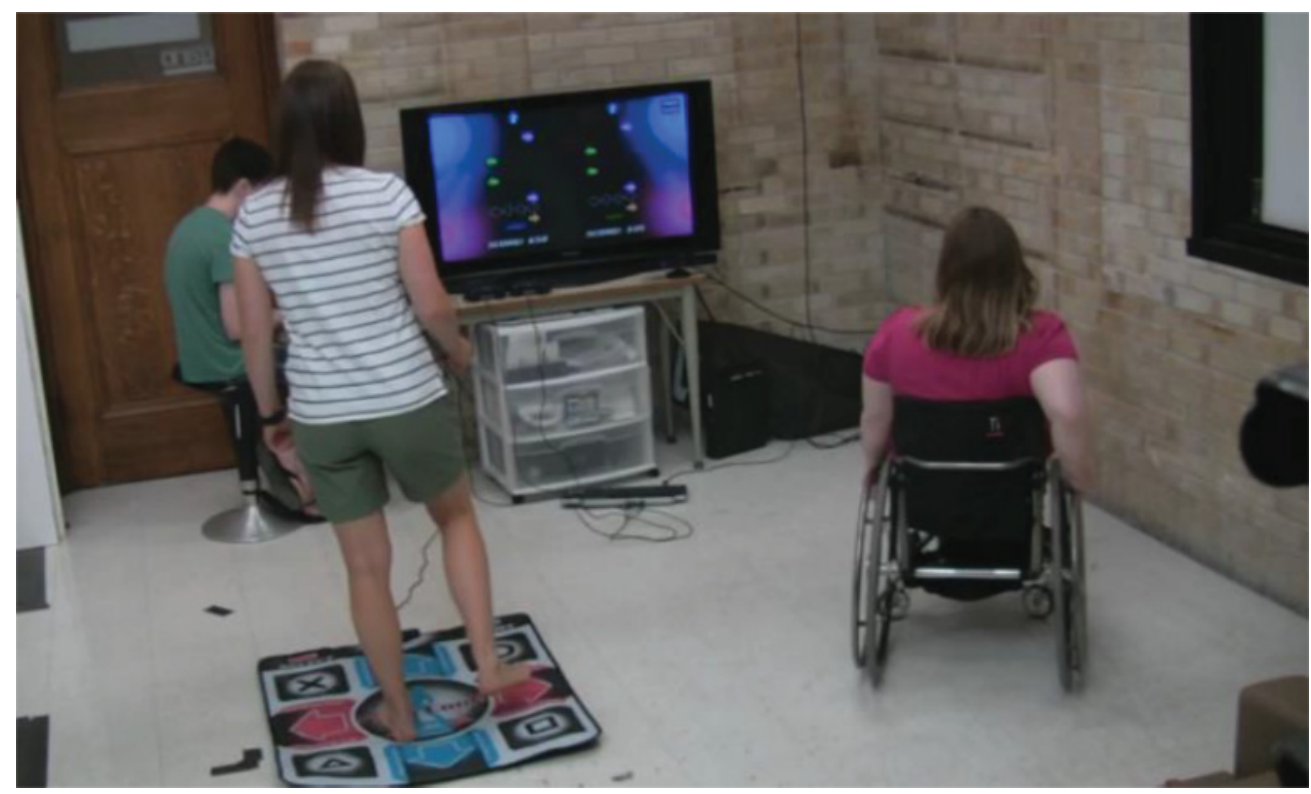

Fig. 3. Two players competing in Wheelchair Revolution.

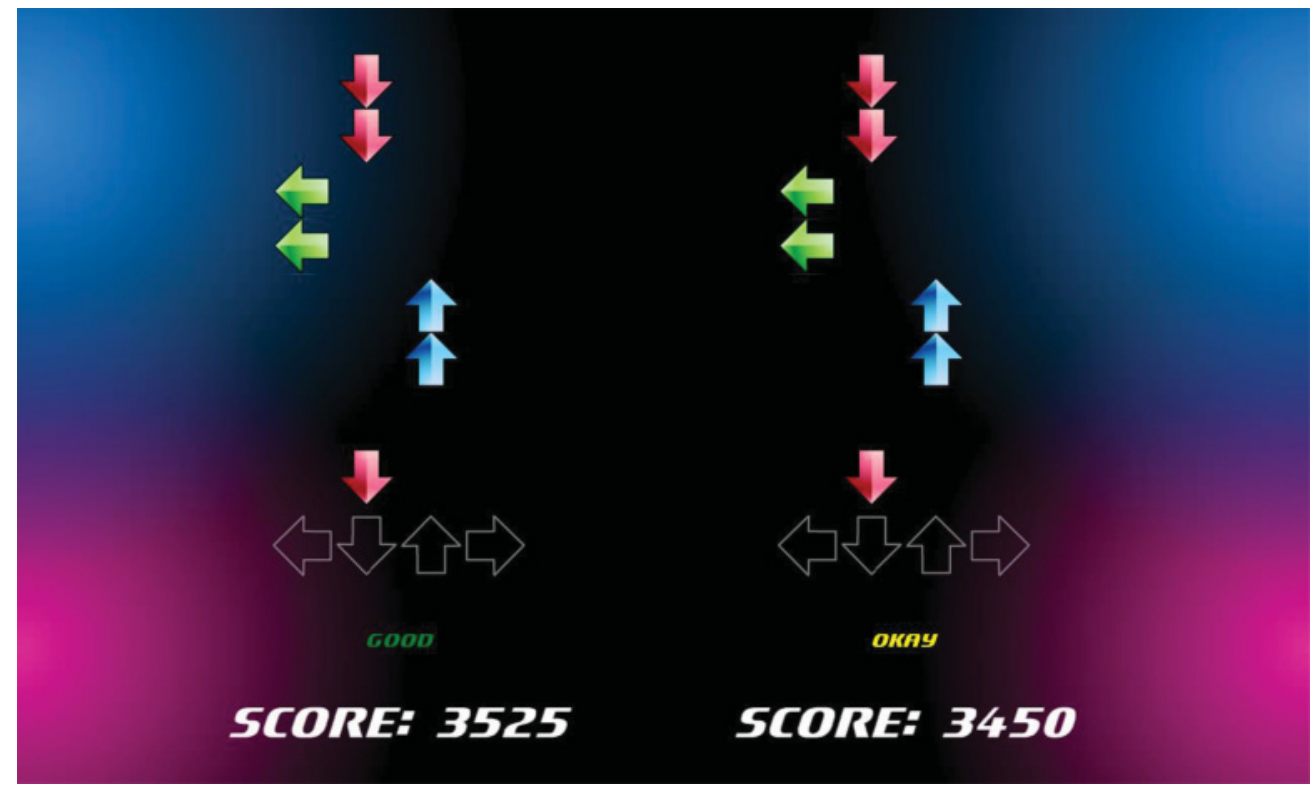

Fig. 4. Screenshot of Wheelchair Revolution.

that closely resembles foot-based input with the goal of providing a comparable player experience.

\subsection{Accommodating Differences in Player Skills and Abilities through Balancing} Building on our initial study with nondisabled persons [Gerling et al. 2014] and recommendations to increase game accessibility by Yuan et al. [2011], the balancing approach 
Table V. Mean (SD) Results for the TLX (20-point scale, composite score 0-120)

\begin{tabular}{llcc}
\hline & \multicolumn{1}{c}{ Item } & Wheelchair Input & Foot-Based Input \\
\hline \multirow{4}{*}{ NASA-TLX } & Composite score & $66.33(4.12)$ & \multicolumn{1}{c}{ Mean (SD) } \\
\cline { 2 - 4 } & Physical demand & $15.00(2.31)$ & $7.67(5.51)$ \\
\cline { 2 - 4 } & Mental demand & $11.67(2.33)$ & $14.33(2.08)$ \\
\cline { 2 - 4 } & Performance & $8.00(3.51)$ & $7.33(3.06)$ \\
\cline { 2 - 4 } & Effort & $13.00(1.73)$ & $13.00(2.00)$ \\
\cline { 2 - 4 } & Frustration & $4.33(3.33)$ & $3.67(2.52)$ \\
\hline
\end{tabular}

applied in Wheelchair Revolution integrates time balancing and score balancing to account for differences between players that may be caused by either the input device (some instances of wheelchair input being slower than body-based input) or individual player skills and abilities. Time balancing adapts time intervals during which players can score perfect moves, thereby mitigating score differences that may be caused by momentum built up in wheelchair input. Likewise, score balancing reduces differences between players by applying individual score multipliers based on initial assessments of player skill.

\subsection{User Study}

We conducted an exploratory user study to evaluate the feasibility of Wheelchair Revolution to provide an opportunity for shared motion-based play with a focus on the accessibility and experience for persons using wheelchairs.

Participants and Procedure. The study was held at the Canadian Paraplegic Association's annual wheelchair relay. Eight persons (four people using wheelchairs and four nondisabled persons) with a mean age of 34 (three female) participated in our study. First, participants provided consent, and participated in an initial round of play to assess their skill level. Second, participants were invited to play two rounds of Wheelchair Revolution. Finally, they were asked to fill in a shortened version of the PENS questionnaire to investigate player experience, asking participants to rate statements regarding dimensions such as perceived competence using 5-point Likert scales [Ryan et al. 2006]. Additionally, we applied the NASA-TLX questionnaire to examine task load [Hart 2006]. Besides player feedback, we included observations and short follow-up interviews in our study.

Results. In this section, we provide an overview of descriptive data as well as qualitative feedback with a focus on the accessibility and player experience that Wheelchair Revolution provides for persons using wheelchairs.

Is wheelchair-based game input suited for persons using wheelchairs, and can it facilitate competition with nondisabled peers? Our results show that all players were able to interact with Wheelchair Revolution on a basic level. Regarding the general accessibility and usability of the system, Table V shows results for the NASA-TLX [Hart 2006], which indicate average levels of overall demand of wheelchair-based input on players, low levels of frustration, and overall levels of demand similar to those players using foot-based input. Interestingly, players using wheelchair input report higher levels of physical demand, while players using foot-based input experienced increased mental demand. In this context, observations revealed that players using wheelchairs had an intuitive understanding of the grid-based interaction paradigm and were able to learn the game controls quickly. When analyzing player scores, we found that persons using wheelchair-based input scored an average of 7061 (SD = 1,277) points for each round of play, which is a promising result regarding the general accessibility of Wheelchair Revolution for persons using wheelchairs. However, in the 


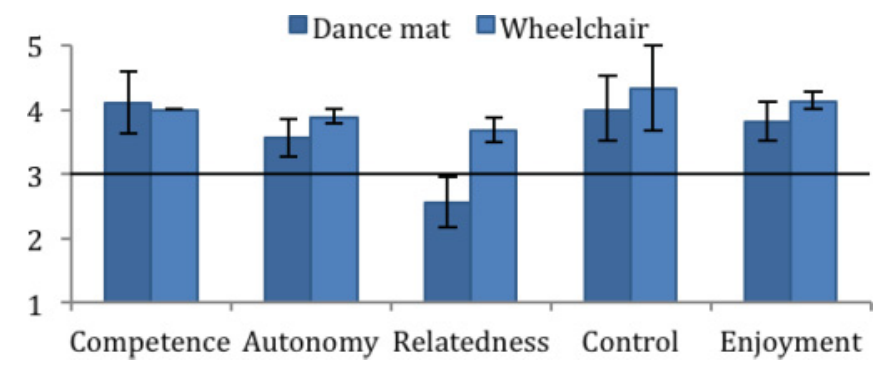

Fig. 5. Mean $( \pm \mathrm{SE})$ player experience scores by input device. The line represents a neutral response.

context of competition between nondisabled persons and persons using wheelchairs, persons using foot-based input scored an average of 10,292 ( $\mathrm{SD}=4,026$ ) points per round, suggesting that balancing intensity needs to be increased to fully accommodate players using wheelchair-based input.

Can wheelchair-based games provide a positive player experience for persons using wheelchairs? Our findings suggest that wheelchair-based games can be a positive experience for persons using wheelchairs. Results of the PENS [Ryan et al. 2006] and IMI [Ryan 1982] questionnaires show that players using wheelchairs reported high levels of enjoyment along with above-average results for all other dimensions (see Figure 5). Interestingly, results for relatedness-investigating how close players feel to others within the context of the game-are raised for persons using wheelchairs, suggesting that the integration of their assistive device in game interaction may, in fact, contribute to a positive overall multiplayer experience. Players perceived their competence with the game as high-regardless of whether they were competing using the wheelchair or dance mat-suggesting that players felt able to master the in-game challenges with the provided input devices. Quotes outline that players were excited about the integration of their wheelchair in the game, stating, for example, that, "it is nice to see my wheelchair in the game instead of being an object that stands between me and the world" (Group 2, person using wheelchair). Similar to findings from study two (Section 4), this suggests that wheelchair-based video games have the potential of providing an empowering, positive player experience that could contribute to the adoption of a positive view of the assistive device as an enabling rather than restricting entity.

How can our results inform the design of future wheelchair-based games to connect players with mobility disabilities with nondisabled peers? On a general level, our results show that motion-based games have great potential to connect persons using wheelchairs and nondisabled persons through physically challenging play. Regarding the experience of players using wheelchairs, our work brings up challenges and opportunities that designers need to consider in future projects. When looking at how players using wheelchairs interacted with the game, we saw some individual differences in terms of movement speed and ability to react to quickly changing in-game challenges, with one player pointing out that, "Coordination is a little hard, but it seems fair." (G3). Likewise, another player pointed out that, "I didn't care if I was losing or winning, I never played the game before." (G2). Considering the fact that none of the participants had had the chance of playing motion-based wheelchair games before, designers should consider the integration of extended tutorial phases to allow players using wheelchairs to familiarize themselves with the input tracking system, its responsiveness, and allow players to adapt their actions to match in-game challenges. In this context, designers need to understand the interesting dual role of the wheelchair as an assistive device and game controller. One participant pointed out that, "If I had 
my sports wheelchair, I would just have to move my arms for the side turns." (G1), suggesting that players understand that the flexibility of their assistive device influences their ability of performing well in the game, ultimately affecting their player experience.

Despite being primarily interested in how the person using wheelchair game input perceived competition, quotes of nondisabled players using foot-based input reveal an interesting design challenge that possibly influences the potential of motion-based games to connect people with mobility disabilities and nondisabled persons. While persons using wheelchairs were not concerned with score differences, for example, pointing out that, "I didn't care if I was losing or winning, I never played the game before" (G4, person using wheelchair), many nondisabled participants were not comfortable beating players using wheelchairs. Quotes of nondisabled participants suggest significant discomfort when beating a person using a wheelchair, with one participant stating that, "I don't think it is fair. We don't seem to be on an equal playing field." (G4, nondisabled person), and another participant outlining that, "[I] felt bad that [the player using the wheelchair] did such a good job and I still beat him" (G2, nondisabled person). While this effect may have been caused by gaps in player scores that could be mitigated by increasing balancing strategies applied in Wheelchair Revolution, the issue needs to be addressed in future game design efforts to ensure that games do not only provide an enjoyable experience for persons using wheelchairs, but also enable nondisabled persons to perceive persons using wheelchairs as competent players.

While more research is necessary to further explore the potential of games to connect nondisabled persons and persons with mobility disabilities, the results of study 3 generally support findings from study 2 , suggesting that wheelchairs can be integrated into motion-based games in an engaging way, and that building on player abilities in the context of motion-based play has the potential of offering an empowering, positive player experience for persons using wheelchairs.

\section{DISCUSSION}

This article investigates wheelchair-based game design in the context of motionbased video games. In the first part of our work, we demonstrate that it is possible to integrate wheelchairs into motion-based game interaction, and we present our toolkit KINECT Wheels. Building on the findings, we present Cupcake Heaven, a wheelchair-based game specifically designed for older adults, and Wheelchair Revolution, a wheelchair-based game that allows persons using wheelchairs to compete with nondisabled players. Our results show that wheelchair-based games are accessible for persons using wheelchairs and provide a positive experience for players of all ages. In the remainder of this article, we discuss the implications of our findings for future work, and show how our results can be applied to increase the quality of life of persons using wheelchairs. Furthermore, we outline how wheelchair-based interaction can increase the accessibility of interactive technologies, and demonstrate the relevance of this work to empower persons with mobility disabilities and foster inclusion through enjoyable shared gaming experiences.

\subsection{Wheelchair-Based Game Controls}

Designing motion-based game controls that allow for the integration of wheelchairs is a promising way of making motion-based video games accessible for people who use wheelchairs. However, there are certain limitations of the presented approach.

Wheelchair-Based Input Gestures. In our research, we demonstrate that wheelchairbased interaction can be applied in an efficient and enjoyable way, which opens up new opportunities for designers, allowing them to integrate wheelchairs as game 
controls. Our evaluation of the gesture set presented in Section 3.1 holds several implications for wheelchair-based interaction design. It shows that it is possible to combine wheelchair-based input and body-based gestures to obtain a range of input gestures broad enough to allow for sufficient complexity in game design. Additionally, results show that completion times differ per gesture, suggesting that designers have to carefully consider the pacing of their games to allow players to transition between gestures (e.g., changing from wheelchair to body input) while also accounting for momentum that wheelchairs build (as our results showed that gestures following wheelchair input are generally slower than sequences of body-based interaction). This issue is particularly interesting when designing for competition between persons using wheelchairs and nondisabled persons: if body-based input is generally faster, game mechanics need to balance speed differences in gesture completion to create a common ground for players of all abilities. Likewise, since wheelchair-based gestures require the use of the hands to move the wheelchair, designers have to take into account that wheelchair-based gestures and hand gestures cannot be performed at the same time. The fact that participants reported that wheelchair-based input was more physically demanding than body-based input, but equally comfortable as body-based gestures suggests that this may be a way of introducing physically challenging motion-based games for persons using wheelchairs.

Technical Limitations. In the design and evaluation of KINECT ${ }^{\text {Wheels }}$ and Cupcake Heaven, we encountered several technical challenges related to the design of camera-based wheelchair interaction. A core challenge during the implementation of KINECT $^{\text {Wheels }}$ was that turning the wheelchair more than 45 degrees caused a loss in recognition. While we were able to achieve acceptable tracking results for turning to the sides through the application of additional algorithms that largely facilitated input recognition for the game Wheelchair Revolution, turning the wheelchair backward or spinning around is still not possible using the current implementation. Additional challenges arise depending on the way designers wish to integrate KINECT ${ }^{\text {Wheels: }}$ because images provided by the Kinect can be noisy, including the absolute position of the wheelchair for precise player input is difficult, and designers should consider including a dead zone similar to joysticks when working with the position of the chair. Likewise, adjustments have to be made if the system is interfacing with other applications using keystrokes; additional algorithms may need to be applied to imitate keyboard input (e.g., repeatedly pressing a button). Finally, moving around the floor space is inherent in wheelchair gestures, and designers have to create games in a way that helps keep the user within the view of the sensor, for example, asking players to move forward multiple times needs to be counteracted with a move backward so that players do not leave the tracking area.

\subsection{Wheelchair Games for Older Adults}

The evaluation of Cupcake Heaven shows that older adults can successfully engage with wheelchair-based video games. This opens up new opportunities for introducing cognitive and physical stimulation to older adults using wheelchairs, particularly care home residents. Our results show that participants not only enjoyed wheelchair-based interaction, but that actively applying the wheelchair as a means of controlling a game also helped them reflect on the way they use their assistive device in daily life. Wheelchair-based games could encourage persons who have only recently started to use a wheelchair to develop a positive relationship with it, helping them to apply it to increase their quality of life rather than regarding it as a limitation to their independence. As a game input device, the wheelchair can be viewed as an enabling technology, rather than a restricting one. In the context of wheelchair-based game design, a challenge that needs to be addressed is adaptive game design for heterogeneous audiences. 
Even among institutionalized older adults living in the same care unit, we observed differences in cognitive and physical abilities, influencing the way these individuals approached the game. These differences extended beyond the basic accessibility of the user interface and general pacing of the game; while simplistic mechanics and a casual approach toward gaming are suitable for some players, they may not keep the interest of others.

Our work shows that adapting design guidelines for motion-based video games for older adults (Section 4.1) is possible to help inform designers wishing to create wheelchair-based games. However, our results also show that wheelchairs can add an additional layer of complexity, calling for careful considerations and balancing of in-game challenges during the design process.

\subsection{Comparing the Experience of Older Adults and Younger Persons}

The evaluations of Cupcake Heaven and Wheelchair Revolution were carried out with fundamentally different participants, with Cupcake Heaven being played by older adults, and Wheelchair Revolution being played by younger persons. While the results suggest that both audiences considered playing wheelchair-based games an enjoyable leisure activity, there are some differences that need to be discussed, regarding the design of the games, the way players interacted with them, and the characteristics of both audiences.

Previous Gaming Experience. A key difference that influenced the experience of older adults and younger adults was previous gaming experience (or the lack thereof). While all younger adults had played video games before, and understood the basic principle of the Kinect controller, older adults required extensive explanations of camerabased game input. Additionally, younger adults were familiar with games similar to Wheelchair Revolution, shaping their expectations, and facilitating their entry into play, whereas many older adults continued to require support to interact with Cupcake Heaven throughout play. While we tried to accommodate these differences through the design of the games-deliberately providing a very simple interaction scheme for Cupcake Heaven-future development efforts should further explore how to best introduce motion-based play to persons without previous gaming experience, for example, through step-by-step tutorials that gradually increase interaction complexity to provide adequate levels of challenge.

Suitability of Interaction Paradigms. Additionally, we observed some differences in physical abilities between older adults and younger persons. While game design for older adults often focuses on the impact of age-related changes, and assumes that older adults are less capable than their younger counterparts, two older adults participating in our study were using wheelchairs on a daily basis, but also maintained their ability to stand and walk short distances, suggesting that games for this demographic might need to consider additional interaction paradigms as proposed within Study 1 (i.e., encouraging players to lift their legs) that may not be suitable for other players using wheelchairs. In contrast, younger adults generally appeared to be more capable when navigating their wheelchairs, regarding both reaction speed and propelling force; together with the fact that younger adults independently navigate their environment using their wheelchair on a daily basis, whereas many older adults in care facilities are supported by nursing staff, the impact of general differences in wheelchair skills as well as physical fitness should be explored to better adapt games to both audiences.

Transitioning into Wheelchair Use versus Long-Term Experience. One final aspect that we believe needs to be considered in the design of wheelchair-controlled video games is the individual history of players in terms of wheelchair use. In our study, 
many older adults were in the process of transitioning into the use of wheelchairs on a daily basis, whereas many younger participants had used wheelchairs for their entire life, or had spent significant periods of time using them. This aspect has implications beyond wheelchair skills: research shows that transitioning into the use of wheelchairs is associated with a range of psychological and emotional challenges [Bates et al. 1993]; therefore, game concepts targeting these audiences need to accommodate individual needs and be aware of vulnerability. While persons with extensive experience in the use of wheelchairs may appreciate games that challenge them to push the limits of their boundaries, these concepts may be frustrating and discouraging for persons who struggle to develop wheelchair skills, or to adapt to their situation. Although games for these audiences should still provide some level of challenge, game designers need to be mindful of individual difficulties, and the potential vulnerability of players, for example, by providing more support to facilitate the entry into play. In this context, future work should explore differences in player experience between persons with a long history of using wheelchairs, and persons having only recently started to use wheelchairs as a result of injury, progressive disease, or age-related changes: only if we understand the needs and challenges that are associated with the use of wheelchairs in a gaming context, will we be able to provide enjoyable and empowering experiences.

\subsection{Wheelchair Games for People with Mobility Disabilities and Nondisabled Persons}

The evaluation of Wheelchair Revolution shows that wheelchair-based game input offers the opportunity of creating motion-based games that are accessible for players of all abilities. Our results show that persons using wheelchairs enjoy applying their wheelchair as a game input device, and report a generally positive player experience in the context of competitive motion-based play with a nondisabled partner. While these results are promising, our findings also suggest that differences in player abilities do influence competition, and the impact of extreme ability differences should be subject to further discussion in the context of game balancing.

Regarding the experience of players using wheelchair input, our results suggest that their experience was not affected by unbalanced competition; however, future research should explore how their experience is affected once novelty effects wear off. To ensure that motion-based play is, in fact, empowering for persons using wheelchairs, effective balancing strategies should be explored further and validated in the context of longterm user studies. Likewise, future research efforts should increase the focus on the nondisabled player. While our work was primarily interested in making motion-based play accessible for persons using wheelchairs, our results provide some evidence that unbalanced competition may have a negative effect on the experience of the nondisabled player. In this context, designing games in a way that allows nondisabled persons to perceive persons with mobility disabilities as physically competent peers is a core challenge in the process of creating positive experiences for nondisabled persons and persons using wheelchairs alike. To address this issue, exploring collaborative rather than competitive motion-based play might be an opportunity for providing enjoyable shared motion-based experiences that allow players of all abilities to work together, focusing on individual player strengths and abilities, rather than risking the further exposition of ability differences.

On a general level, the results of our three studies demonstrate that wheelchair-based video games can be designed to be accessible for persons using wheelchairs. Despite differences in abilities of navigating wheelchairs as well as the impact of age-related changes and impairments, both older and younger adults perceived the integration of the wheelchair as a game controller as enjoyable and empowering, which highlights 
the large potential of wheelchair-based video games to provide a physically stimulating leisure activity.

\section{DESIGN OPPORTUNITIES}

Based on the work presented in this article, we identified areas in which wheelchairbased interaction can be applied in a way that can contribute to quality of life of persons with mobility disabilities. In this section, we discuss the three most promising opportunities.

\subsection{Wheelchair Skills Training}

The development of wheelchair skills is a crucial step in encouraging older adults to embrace their assistive device and maintain a high degree of functional independence. However, many older adults only start using a wheelchair late in life, and experience difficulties in developing a positive relationship with it. Wheelchair skills training is a promising opportunity that has been shown to be effective with younger age groups [Sawatzky et al. 2012]. Older adults are encouraged to participate in occupational therapy to become familiar with their wheelchair; however, this requires access to a therapist and can be tedious. Wheelchair-based game controls could provide in-home wheelchair skills training; if input gestures are designed to support therapy goals, game-based interventions could help older adults become more familiar with their wheelchair. In addition, motivational aspects of video game play could be leveraged to provide wheelchair skills training for younger audiences, for example, children and teenagers; in this context, future work should explore the development of more complex game concepts that appeal to players who are generally familiar with video games and that could further encourage the adoption of wheelchair skills. In general, such games could help players' abilities of navigating their environment, increase their functional independence, and thereby improve their quality of life.

\subsection{Games to Facilitate Physical Activity to Promote Healthy Lifestyles}

Another opportunity that is inherent to movement-based play is the facilitation of physical activity to promote healthy lifestyles. A large body of research has demonstrated the effectiveness of motion-based games or exergames in providing moderate-to-vigorous intensity level exercise for nondisabled persons (e.g., Gao and Mandryk [2011]), suggesting that such games are a suitable means of providing physical activity. In the context of wheelchair-based game design, creating games that not only implement movement-based input, but actually encourage significant energy expenditure among players could be a valuable means of providing exercise for an audience that is often sedentary due to the lack of wheelchair-accessible activities [U.S. Department of Health and Human Services 2010]. While the work presented in this article has mostly focused on interface-related challenges and aspects of entertainment, it paves the way for future work that could explore whether KINECT Wheels [Gerling et al. 2013] can be integrated into wheelchair-based exergames as a means of providing in-home exercise for persons using wheelchairs, for example, by investigating levels of energy expenditure and other health outcomes that may be improved by such games, functional requirements of wheelchair-based exergames using our toolkit, and considerations regarding long-term player motivation and engagement to support players wishing to improve their fitness.

\subsection{Games to Foster Inclusion and Empower Persons Using Wheelchairs}

Video games are an effective means of promoting positive relationships among players [De Kort and IJsselsteijn 2009]. Our work shows that it is possible to engage nondisabled persons and persons with mobility disabilities in motion-based play. In 
this context, we believe that leveraging the positive effects of video games as a shared activity offers the opportunity of fostering the inclusion of persons with mobility disabilities. Especially with respect to the physical dimension of motion-based play, such games offer two main opportunities: First, motion-based video games, in general, can empower persons using wheelchairs to apply their assistive device in an enjoyable context, and experience physical competence through successfully overcoming in-game challenges in motion-based play. Second, shared motion-based games, in particular, allow nondisabled persons to understand the strengths and abilities of persons using wheelchairs, ultimately enabling nondisabled persons to perceive persons using wheelchairs as physically competent peers, which could contribute to disability awareness and the promotion of positive attitudes toward people with disabilities.

\section{CONCLUSION}

Our work can help designers to build upon the abilities of players using wheelchairs rather than making limited subsets of existing motion-based interaction paradigms accessible. Because of the increasing popularity of motion-based video games, it is important to encourage the inclusion of wheelchair-accessible control schemes to allow persons using wheelchairs to participate in play and obtain the full benefits of motionbased video games. In that context, we believe that wheelchair-based video games are a valuable opportunity to encourage physical and cognitive activity among broad audiences ranging from children and teenagers using wheelchairs to older adults. Besides the immediate benefits of playing motion-based video games, the integration of wheelchair movements has the potential to improve an individual's relationship with their assistive device and increase their abilities of using wheelchairs to independently navigate their environment. Furthermore, our work highlights the potential of shared motion-based play to connect persons using wheelchairs with nondisabled peers, allowing them to experience physical competence in the context of motion-based games, and encouraging them to view their assistive deice as an enabling entity, thereby contributing to their quality of life.

\section{ACKNOWLEDGMENTS}

We thank Saskatoon Health Region, Sherbrooke Community Centre, Brightwater Senior Living, and the Canadian Paraplegic Association for supporting our work, and the participants for their contribution.

\section{REFERENCES}

R. Aarhus, E. Groenvall, S. B. Larsen, and S. Wollsen. 2011. Turning training into play: Embodied gaming, seniors, physical training and motivation. Gerontechnology 10, 2 (2011), 110-120.

P. S. Bates, J. C. Spencer, M. E. Young, and D. H. Rintala. 1993. Assistive technology and the newly disabled adult: Adaptation to wheelchair use. Am. J. Occup. Ther. 47 (1993), 1014-1021.

S. Cuzzort, and T. Starner. 2008. AstroWheelie: A wheelchair-based exercise game. In IEEE Proceedings of ISWC'08. 113-114.

S. J. Czaja and C. C. Lee. 2006. Information technology and older adults. In The Human-Computer Interaction Handbook. A. Sears and J. A. Jacko, (Eds.). Lawrence Earlbaum Associates, New York and London.

Y. A. W. De Kort and W. A. IJsselsteijn. 2009. People, places, and play: Player experience in a socio-spatial context. Comput. Entertain. 6, 2 (2009).

S. A. Douglas, A. E. Kirkpatrick, and I. S. MacKenzie. 1999. Testing pointing device performance and user assessment with the ISO 9241, Part 9 standard. In Proceedings of CHI '99. ACM, New York, NY, 215-222.

T. Felzer and B. Freisleben. 2002. HaWCoS: The "hands-free" wheelchair control system. In Proceedings of ASSETS '02. ACM, New York, NY, 127-134.

Y. Gao and R. L. Mandryk. 2011. GrabApple: The design of a casual exergame. In Proceedings of the 10th International Conference on Entertainment Computing. Springer, 35-46.

K. M. Gerling, M. R. Kalyn, and R. L. Mandryk. 2013. KINECTWheels: Wheelchair-accessible motion-based game interaction. In Proceedings of CHIEA'13. ACM, New York, NY, 3055-3058. 
K. M. Gerling, I. J. Livingston, L. E. Nacke, and R. L. Mandryk. 2012. Full-body motion-based game interaction for older adults. In Proceedings of CHI 2012. ACM, New York, NY, 1873-1882.

K. M. Gerling, R. L. Mandryk, and M. R. Kalyn. 2013. Wheelchair-based game design for older adults. In Proceedings of ASSETS '13. ACM, New York, NY, Article 27.

K. M. Gerling, M. Miller, R. L. Mandryk, M. Birk, and J. Smeddinck. 2014. Effects of balancing for physical abilities on player performance, experience and self-esteem in exergames. In Proceedings of CHI 2014. ACM, New York, NY, 2201-2210.

S. G. Hart. 2006. NASA-task load index (NASA-TLX); 20 years later. In Proceedings of the Human Factors and Ergonomics Society 50th Annual Meeting.

A. Hasdai, A. S. Jessel, and P. L. Weiss. 1998. Use of a computer simulator for traning children with disabilities in the operation of a powered wheelchair. Am. J. Occup. Ther. 52, 3 (1998).

J. B. Hinkel. 2010. Head-guided wheelchair control system. In Proceedings of ASSETS '10. ACM, New York, NY, 313-314.

J. Höysniemi. 2006. International survey on the Dance Dance Revolution game. Comp. Entertain. 4, 2 (2006),

Y. Jung, K. J. Li, N. S. Janissa, W. L. C. Gladys, and K. M. Lee. 2009. Games for a better life: Effects of playing Wii games on the well-being of seniors in a long-term care facility. In Proceedings of IE '09. ACM, New York, NY, Article 5.

E. Ketcham and G. E. Stelmach. 2001. Age-related declines in motor control. In Handbook of the Psychology of Aging, J. E. Birren and K. W. Schaie (Eds.). Academic Press, San Diego, CA.

P. Keyani, G. Hsieh, B. Mutlu, M. Easterday, and J. Forlizzi. 2005. DanceAlong: Supporting positive social exchange and exercise for the elderly through dance. In Proceedings of CHIEA'05. ACM, New York, NY, 1541-1544.

R. L. Mandryk, K. M. Gerling, and K. S. Stanley. 2014. Designing games to discourage sedentary behaviour. In Playful User Interfaces, Gaming Media and Social Effects, A. Nijholt (Ed.). Springer.

T. J. O’Connor, R. A. Cooper, S. G. Fitzgerald, M. J. Dvorznak, M. L. Boninger, D. P. VanSickle, and L. Glass. 2000. Evaluation of a manual wheelchair interface to computer games. Neurorehabil. Neural Repair 14 , 21 (2000).

M. Rice, M. Wan, M.-H. Foo, J. Ng, Z. Wai, J. Kwok, S. Lee, and L. Teo. 2011. Evaluating gesture-based games with older adults on a large-screen display. In Proceedings of Sandbox'11. ACM, New York, NY, $17-24$.

J. Rimmer, B. Riley, E. Wang, A. Rauworth, and J. Jurowski. 2004. Physical activity participation among persons with disabilities: Barries and facilitators. Am. J. Prev. Med. 26, 5 (2004), 419-425.

N. Rossol, I. Cheng, W. F. Bischof, and A. Basu. 2011. A framework for adaptive training and games in virtual reality rehabilitation environments. In Proceedings of VRCAI 2011. ACM, New York, NY, 343-346.

R. M. Ryan. 1982. Control and information in the intrapersonal sphere: An extension of cognitive evaluation theory. J. Pers. Soc. Psychol. 43 (1982), 450-461.

R. M. Ryan, C. S. Rigby, and A. K. Przybylski. 2006. Motivational pull of video games: A self-determination theory approach. Motiv. Emotion 30 (2006).

B. Sawatzky, P. W. Rushton, I. Denison, and R. McDonald. 2012. Wheelchair skills training programme for children: A pilot study. Aust. Occup. Ther. J. 59 (2012).

M. S. Tremblay, R. Colley, T. J. Saunders, G. N. Healy, and N. Owen. 2010. Physiological and health implications of a sedentary lifestyle. Appl. Physiol. Nutr. Metab. 35, 6 (2010), 725-740.

U. S. Department of Health and Human Services. 2010. Healthy people 2010. Conference edition, 2 (2010), U. S. Department of Health and Human Services, Washington, DC.

M. A. Vidulich and P. S. Tsang. 1986. Collecting NASA workload ratings: A paper and pencil package. Working paper. NASA Ames Research Center, Moffett Field, CA.

J. O. Wobbrock, B. A. Myers, H. H. Aung, and E. F. LoPresti. 2004. Text entry from power wheelchairs: EdgeWrite for joysticks and touchpads. In Proceedings of ASSETS '04. ACM, New York, NY, 110-117.

W. Young, S. Ferguson, S. Brault, and C. Craig. 2011. Assessing and training standing balance in older adults: A novel approach using the "Nintendo Wii" balance board. Gait Posture 33, 2 (2011).

B. Yuan, E. Folmer, and F. Harris. 2011. Game accessibility: A survey. Univ. Access Inf. Soc. 10 (2011), 81-100.

Received June 2014; revised January 2015; accepted January 2015 